\title{
Guidelines for the management of iron deficiency anaemia
}

\author{
A F Goddard, A S McIntyre, B B Scott, for the British Society of Gastroenterology
}

\begin{abstract}
Summary
Iron deficiency anaemia in men and postmenopausal women is most commonly caused by gastrointestinal blood loss or malabsorption. Examination of both the upper and lower gastrointestinal tract is therefore an important part of the investigation of patients with such anaemia. In the absence of overt blood loss or any obvious cause, all patients should have upper gastrointestinal endoscopy, including small bowel biopsy, and colonoscopy or barium enema to exclude gastrointestinal malignancy. Further gastrointestinal investigation is only warranted in transfusion dependent anaemia or where there is visible blood loss. Treatment of an underlying cause will cure the anaemia but even when no cause is detected the long term outlook is good.
\end{abstract}

\subsection{Introduction}

Iron deficiency anaemia (IDA) occurs in $2-5 \%$ of adult men and post-menopausal women in the developed world ${ }^{12}$ and is a common cause of referral to a gastroenterology clinic (4-13\% of referrals). ${ }^{3}$ While menstrual blood loss is the commonest cause of IDA in pre-menopausal women, blood loss from the gastrointestinal (GI) tract is the commonest cause in adult men and post-menopausal women. ${ }^{4-8}$ Asymptomatic colonic and gastric carcinoma may present with IDA and exclusion of these conditions is of prime concern. Malabsorption (most frequently from coeliac disease), poor dietary intake, previous gastrectomy, and NSAID use are not unusual but there are many other possible causes (table 1). The management of IDA is often suboptimal with most patients being incompletely investigated if at all. ${ }^{9}$

These guidelines are primarily for gastroenterologists and GI surgeons but would be applicable to other doctors seeing patients with IDA.

The investigation of overt blood loss is not considered in these guidelines.

\subsection{Definitions}

2.1 ANAEMIA

The diagnostic criteria for anaemia in IDA vary $(\mathrm{Hb}<10-11.5 \mathrm{~g} / \mathrm{dl}$ for women and $<12.5-13.8$ $\mathrm{g} / \mathrm{dl}$ for men) between studies. The lower limit of the normal range of haemoglobin concentration for the laboratory performing the test should therefore probably be used to define anaemia ${ }^{\star}$. It is not known at what level of haemoglobin investigations should be initiated. However, there is no a priori reason why mild anaemia should be less indicative of important disease than severe anaemia.
Table 1 Gastrointestinal (GI) diseases presenting with iron deficiency

\begin{tabular}{l} 
Occult GI blood loss \\
\hline Common \\
NSAID use \\
Colonic cancer/polyp \\
Gastric cancer \\
Angiodysplasia \\
Crohn's disease \\
Ulcerative colitis \\
Uncommon \\
Oesophagitist \\
Peptic ulcert \\
Oesophageal cancer \\
Water melon stomach \\
Intestinal telangiectasia \\
Lymphoma, leiomyoma and other small bowel tumours \\
Duodenal polyp (Brunner's gland adenoma) \\
Carcinoma of the ampulla of Vater \\
Meckel's diverticulum \\
Hookworm \\
Malabsorption \\
Coeliac disease \\
Gastrectomy (partial and total) and gastric atrophy \\
Gut resection or bypass \\
Bacterial overgrowth \\
Whipple's disease \\
Lymphangiectasia
\end{tabular}

†Although common causes of acute bleeding they are uncommon causes of occult bleeding.

2.2 IRON DEFICIENCY (ID)

Microcytosis (mean corpuscular volume (MCV) lower than the normal range) is characteristic of ID but it may also occur in much less common conditions such as thalassaemia (when the red cell count is usually elevated). Haemoglobinopathies frequently cause microcytosis in certain ethnic groups but this should not be presumed to be the cause unless confirmed by laboratory testing. Microcytosis may be absent in combined deficiency (e.g. with folate deficiency) which may be recognised by a raised red cell distribution width (RDW). The anaemia of chronic disease due to the inability to use iron may also present with microcytosis.

Serum ferritin concentration is the most powerful test for ID ${ }^{\star \star \star} \cdot{ }^{10}$ A serum ferritin concentration of $<12 \mu \mathrm{g} / \mathrm{dl}$ is diagnostic of ID ${ }^{\star \star} .{ }^{11}$ However, serum ferritin may be raised above $12-15 \mu \mathrm{g} / \mathrm{dl}$ in patients with ID and concurrent chronic inflammation, malignancy, or hepatic disease, although if the concentration is $>100 \mu \mathrm{g} / \mathrm{dl}$, ID is almost certainly not present.

A further test is usually only required in patients when doubt still remains as to the presence of iron deficiency $\star \star 1112$ and advice from a haematologist should be sought. Red cell protoporphyrin concentration and

Abbreviations used in this paper: ID, iron deficiency; IDA, iron deficiency anaemia; RDW, red cell distribution width; GI, gastrointestinal; MCV, mean corpuscular volume. 
- Serum ferritin concentration is the most powerful test for iron deficiency ${ }^{\star \star \star}$.

transferrin saturation of $<30 \%$ may help the diagnosis but a therapeutic response to three weeks of oral iron or a bone marrow aspiration are the only ways of confirming true deficiency ${ }^{\star \star \star} .{ }^{10}$ New tests which involve measuring the serum transferrin binding receptor/ ferritin ratio show promise in distinguishing between anaemia of chronic disease and iron deficiency but are not yet widely available.

The need for investigation of patients with iron deficiency but no anaemia has not been assessed in clinical studies.

\subsection{Investigations}

3.1 HISTORY

A careful dietary history is important to identify iron deficient diets. However, since borderline deficient diets are common in patients, a positive dietary history should not be presumed as the cause of anaemia and a full GI investigation is still required. The presence of upper and lower GI symptoms should be documented although these rarely correlate with investigation findings. The use of aspirin and analgesics, particularly NSAIDs, should be noted and stopped whenever possible. The use of these drugs and anticoagulants should not usually deter investigation. Family history of haematological disorders (for example thalassaemia and sideroblastic anaemia), telangiectasia, and bleeding disorders should be sought.

\subsection{EXAMINATION}

Careful examination, although seldom contributory, may reveal a relevant abdominal mass or cutaneous signs of gastrointestinal blood loss (for example Peutz-Jeghers and Osler-Weber-Rendu syndromes).

3.3 GASTROINTESTINAL EVALUATION

GI investigations should be considered in all patients in whom IDA has been confirmed unless there is a history of significant non-GI blood loss. In the absence of suggestive symptoms the order of investigations will be determined by local availability. It is usually convenient to do upper GI endoscopy first although in the elderly investigation of the colon is likely to be more productive. Upper GI endoscopy can be expected to reveal a cause in between 30 and $50 \%$ of patients. ${ }^{4-8}$ Small bowel biopsies should be taken during this endoscopy as $2-3 \%$ of patients presenting with IDA have coeliac disease ${ }^{\star \star} .{ }^{34}$ If the patient is unable to have upper GI endoscopy, a barium meal should be performed in addition to blood antiendomysial antibody.

- Small bowel biopsies should be taken during endoscopy as $2-3 \%$ of patients presenting with iron deficiency anaemia have coeliac disease ${ }^{\star \star}$.
Unless the upper GI endoscopy reveals carcinoma or coeliac disease, all patients should then undergo examination of the lower GI tract ${ }^{\star \star \star 1}{ }^{3-8}$ as dual pathology (lesions in both the colon and upper GI tracts) occurs in around $10-15 \%$ of patients. In particular, oesophagitis, erosions, aphthous ulceration, and peptic ulcer should not at this stage be accepted as the cause of the iron deficiency. Colonoscopy (possibly at the same session as the upper GI endoscopy-'bidirectional endoscopy') has the advantage that it will demonstrate angiodysplasia and allow biopsy of any lesion. However, double contrast barium enema is a sufficient alternative, ${ }^{13}{ }^{14}$ with or without sigmoidoscopy ${ }^{13}$ especially if the facilities for colonoscopy are limited or the success rate of complete colonoscopy is poor within a particular unit. Omission of sigmoidoscopy appears safe if digital rectal examination is negative in the absence of a changed bowel habit or rectal bleeding. ${ }^{13}$

\subsection{FURTHER EVALUATION}

Further direct visualisation of the small bowel is probably not necessary unless the IDA is transfusion dependent or there has been visible blood loss ${ }^{\star \star} \cdot{ }^{37}$ Follow-up studies have shown this approach to be safe ${ }^{13} 15$ provided dietary deficiency is corrected and/or NSAIDs stopped and the haemoglobin concentration monitored. However, if IDA is transfusion dependent, enteroscopy may be helpful to detect and treat small bowel angiodysplasia ${ }^{\star \star} .{ }^{16}$ Small bowel radiology is rarely of use unless the history is suggestive of Crohn's disease ${ }^{\star \star} .{ }^{5} 18$ Mesenteric angiography is of limited use but may be valuable in transfusion dependent IDA for demonstrating vascular malformations. Similarly, diagnostic laparotomy with on-table endoscopy may be considered in cases which have defied other investigation but is unlikely to be resorted to unless there is transfusion dependent anaemia ${ }^{2}$. Meckel's diverticulum usually presents with visible blood loss (melaena) but may rarely present with IDA and should be considered in young adults. Diagnostic laparotomy is the most sensitive test for Meckel's diverticulum while pertechnicate scans have very poor sensitivity ${ }^{\star \star} .{ }^{19}$

Other investigations, including routine assessments of the liver and renal function, and clotting studies are of no diagnostic value unless the history is suggestive of systemic disease ${ }^{\star \star} .{ }^{3}$ Faecal occult blood testing is of no benefit in the investigation of IDA, being insensitive and non-specific ${ }^{\star \star} .{ }^{40}$ Very occasionally, urinary tract tumours may present with IDA and therefore the presence of haematuria should be excluded.

\subsection{Management}

4.1 AIM OF TREATMENT

The aim of treatment should be to restore haemoglobin levels and MCV to normal and replenish body stores. If this cannot be achieved, consideration should be given to further evaluation. 


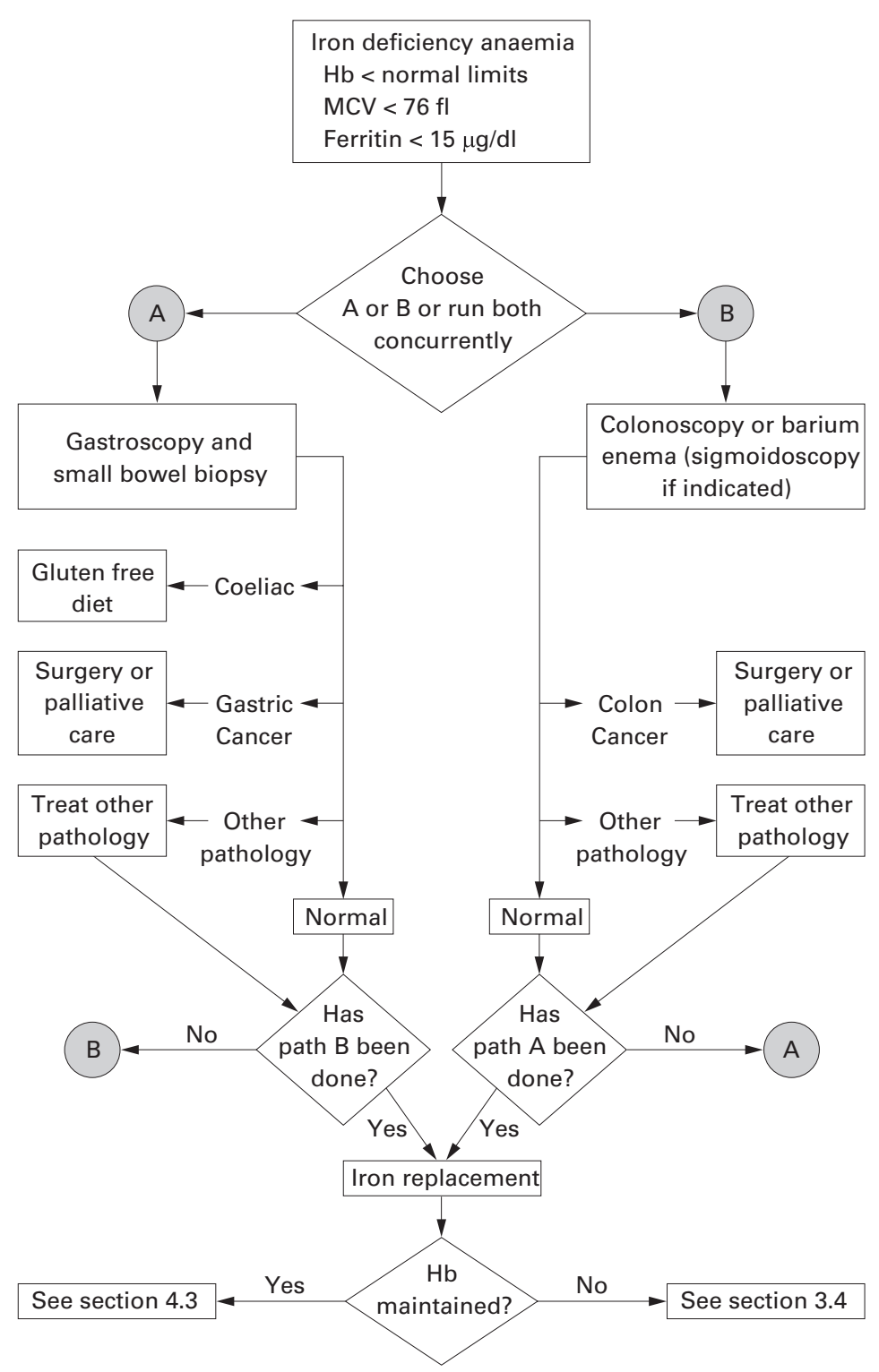

Figure 1 Flow chart for the management of iron deficiency anaemia in men and non-menstruating women (for other patient groups see text).

\subsection{IRON THERAPY}

Treatment of an underlying cause should prevent further iron loss but all patients should have iron supplementation both to correct anaemia and replenish body stores ${ }^{\star \star} .{ }^{21}$ This is achieved most simply and cheaply with ferrous sulphate $200 \mathrm{mg}$ three times daily although ferrous gluconate and ferrous fumarate are as effective. ${ }^{21}$ A liquid preparation may be tolerated when tablets are not. Ascorbic acid enhances iron absorption ${ }^{\star \star 22}$ and should be considered when response is poor. Parenteral iron should only be used when there is intolerance to at least two oral preparations or non-compliance. Parenteral iron treatment is painful (when given intramuscularly), expensive, and may cause anaphylactic reactions. The rise in haemoglobin is no quicker than with oral preparations. The haemoglobin concentration should rise by $2 \mathrm{~g} / \mathrm{dl}$ after $3-4$ weeks. Failure to do so is usually due to poor compliance, misdiagnosis, continued blood loss, or malabsorption. Iron supplementation should be continued for three months after correction of anaemia to replenish iron stores $^{\star}{ }^{21}$

- All patients should receive iron supplementation to correct anaemia and replenish body stores ${ }^{\star \star}$.

4.3 FOLLOW UP

Once normal, the haemoglobin concentration and red cell indices should be monitored at intervals. We suggest three monthly for one year and then after a further year. Additional oral iron should be given if the haemoglobin or MCV falls below normal (a ferritin estimation should also be done in doubtful cases). Further investigation is only necessary if the haemoglobin and MCV cannot be maintained in this way. It is reassuring to know that iron deficiency does not return in most patients in whom a cause for IDA is not found after upper GI endoscopy, small bowel biopsy and barium enema. ${ }^{13}$

\subsection{SUMMARY FLOW CHART}

A management flow chart is shown in fig 1 .

\subsection{Special considerations}

5.1 CO-MORBIDITY

The appropriateness of investigating patients with severe co-morbidity or other reason (in some circumstances advanced age) especially if the result would not influence management, should be carefully considered and discussed with patients and carers when possible.

\subsection{PRE-MENOPAUSAL WOMEN}

Menstruating women present a large healthy population in which IDA is common, occurring in $5-10 \% .{ }^{23}$ Menstrual loss, especially menorrhagia, pregnancy, and breast feeding are usually responsible. ${ }^{24}$ History is unreliable in quantifying menstrual loss, ${ }^{25}$ although pictorial blood loss assessment charts have been shown to have a sensitivity and specificity of around $80 \%$ for detecting menorrhagia. ${ }^{26}$ There are little data on the yield of GI investigation in menstruating women with IDA $^{27} 28$ but significant GI pathology has been detected in these studies.

- Iron deficiency anaemia occurs in $5-10 \%$ of menstruating women.

Because of the increasing incidence of important pathology with age, we recommend that those more than 45 years are investigated according to the above guidelines. In the absence of data in those less than 45 years, we recommend that only patients with upper GI symptoms have endoscopy and small bowel biopsy. The remainder should have antiendomysial antibody determinations (and IgA measurement to exclude IgA deficiency which makes the test unreliable) to exclude coeliac disease. Colonic investigation in patients less than 45 years should only be done if there are 
colonic symptoms, a strong family history of colorectal carcinoma (for example two first degree relatives or one first degree relative $<45$ years), or persistent IDA following iron supplementation and correction of potential causes of losses (for example menorrhagia, blood donation, poor diet).

5.3 YOUNG MEN

Although the incidence of important GI pathology in young men is low, there are no data on the yield of investigation in those with IDA. In the absence of such data we recommend that investigation of young men should occur according to the guidelines.

5.4 POST-GASTRECTOMY

IDA is to be expected after gastrectomy, both partial and total, ${ }^{29}$ due to poor chelation and absorption of iron as a result of the loss of ascorbic acid and hydrochloric acid, and loss of free iron in exfoliated cells. It would seem reasonable, therefore, only to investigate those whose IDA persists on iron supplementation or who present with IDA many years after partial gastrectomy.

\subsection{Suggested targets for audit}

We suggest that:

- $90 \%$ of patients (other than menstruating women) with iron deficiency anaemia and no obvious cause should have both an upper GI endoscopy with small bowel biopsy and either colonoscopy or barium enema (unless a firm cause is found with the first investigation).

- Resolution of anaemia should be achieved by six months in $80 \%$ of patients.

- $90 \%$ of those not responding to treatment should have been considered for further investigation.

\subsection{Suggested topics for further research}

- The need to investigate iron deficiency without anaemia.

- The importance of stratifying for risk of significant disease according to haemoglobin level.

- The long term prognosis of colonic neoplasms discovered by investigating iron deficiency anaemia compared with those found in other ways.

- The role of investigation in young men and menstruating women.

- The value of CT colonography in the investigation of IDA.

\subsection{Strength of recommendations made in these guidelines}

The strength of evidence for recommendations given in these guidelines is indicated by ${ }^{\star}, \star \star$, or $\star \star \star \star$ in the text as follows:

$\star \star \star$ Based on meta-analysis or large randomised trials.

${ }^{\star}$ Based on good evidence from trials, but less convincing (e.g. smaller numbers).

${ }^{\star}$ Based on specialist opinion.

\subsection{Formulation of guidelines}

These guidelines were drawn up by the following process:

(1) Medline search using the term "iron deficiency anaemia".

(2) Review of abstracts of all references.

(3) Review of all papers felt to be relevant to guidelines.

(4) Review of references not cited by Medline felt to be relevant to guidelines.

(5) Drafts of guidelines formulated by authors.

(6) Initial guidelines reviewed by BSG Clinical Services and Standards Committee.

(7) Guidelines amended by authors.

(8) Steps (6) and (7) repeated twice.

(9) Guidelines accepted by BSG Clinical Services and Standards Committee.

(10) Guidelines reviewed by BSG council.

(11) Guidelines amended by authors.

(12) Guidelines accepted by council and submitted for publication.

\subsection{Date for review}

April 2004.

\subsection{References}

1 Calvey HD, Castleden CM. Gastrointestinal investigations for anaemia in the elderly: a prospective study. Age Ageing 1987;16:399-404

2 Sayer JM, Long RG. A perspective on iron deficiency anaemia. Gut 1993;34:1297-9.

3 McIntyre AS, Long RG. Prospective survey of investigations in outpatients referred with iron deficiency anaemia. Gut 1993;34:1102-7.

4 Kepczyk T, Kadakia SC. Prospective evaluation of gastrointestinal tract in patients with iron-deficiency anemia. Dig Dis Sci 1995;40:1283-9.

5 Rockey DC, Cello JP. Evaluation of the gastro-intestinal tract in patients with iron-deficiency anemia. $N$ Engl f Med 1993;329:1691-5.

6 Cook IJ, Pavli P, Riley JW, Goulston KJ, Dent OF. Gastrointestinal investigation of iron deficiency anaemia. Gastrointestinal investigation of iron deficiency anaemia.

7 Zuckerman G, Benitez J. A prospective study of bidirectional endoscopy (colonoscopy and upper endoscopy) in the evaluation of patients with occult gastrointestinal bleeding. Am 7 Gastroenterol 1992;87:62-6.

8 Hardwick RH, Armstrong CP. Synchronous upper and lower gastrointestinal endoscopy is an effective method of investigating iron-deficiency anaemia. Br f Surg 1997;84: $1725-8$

9 Lucas CA, Logan ECM, Logan RFA. Audit of the investigation and outcome of iron-deficiency anaemia in one health district. $\mathcal{F} R$ Coll Physicians Lond 1996;30:33-5.

10 Guyatt GH, Oxman AD, Ali M, Willan A, McIlroy W, Patterson C. Laboratory diagnosis of iron-deficiency anemia: an overview. f Gen Intern Med 1992;7:145-53.

11 Cook JD, Baynes RD, Skikne BS. Iron deficiency and the measurement of iron status. Nutr Res Rev 1992;5:189-202.

12 Doube A, Davis M, Smith JG, Maddison PJ, Collins AJ. Structured approach to the investigation of anaemia in
patients with rheumatoid arthritis. Ann Rheum Dis patients with rhe

13 Sahay R, Scott BB. Iron deficiency anaemia-how far to investigate? Gut 1993;34:1427-8.

14 Sayer JM, Donnelly MT, McIntyre AS, et al. Is colonoscopy necessary as a first line investigation in iron deficiency anaemia. Gut 1995;36(suppl II):A35.

15 Gordon S, Bensen S, Smith R. Long-term follow up of older patients with iron-deficiency anemia after a negative GI evaluation. Am f Gastroenterol 1996;91:885-9.

16 Davies GR, Benson MJ, Gertner DJ, van Someren RMN, Rampton DS, Swain CP. Diagnostic and therapeutic push type enteroscopy in clinic use. Gut 1995;37:346-52.

17 Morris AJ, Wasson LA, MacKenzie JF. Small bowel enteroscopy in undiagnosed gastrointestinal blood loss. Gut 1992;33:887-9.

18 Till SH, Grundman MJ. A prospective audit of patients presenting with iron deficiency anaemia and faecal occult blood loss. Gut 1992;33(suppl 1):S31. 
19 Thompson JN, Salem RR, Hemingway AP, et al. Specialist investigation of obscure gastrointestinal bleeding. Gut investigation of

20 Moses PL, Smith RE. Endoscopic evaluation of iron deficiency anaemia. Postgrad Med 1995:98:213-26.

21 Smith AG. Prescribing iron. Prescribers' $\mathcal{F} 1997 ; 37: 82-7$.

22 Hallerg L, Brune M, Rossander-Hulthen R. Is there a physiological role of vitamin C in iron absorption. Ann NY Acad Sci 1987;498:324-32.

23 World Health Organisation. The prevalence of anaemia in women: A tabulation of available information, 2 nd edn. Geneva: World Health Organisation, 1992.

24 Allen LH. Pregnancy and iron deficiency: unresolved issues. Nutr Rev 1997;55:91-101.
25 McKenna DM, Dockeray CJ, McCann SR. Iron deficiency in pre-menopausal females. Ir Med F 1989;82:69-70.

26 Higham JM, O'Brien PM, Shaw RW. Assessment of menstrual blood loss using a pictorial chart. Br $\mathcal{F}$ Obstet Gynaecol 1990;97:734-9.

27 Sayer JM, Donnelly MT, Ching CK, Long RG. The aetiology of iron deficiency anaemia in pre-menopausal women. Gastroenterology 1994;106:A26.

28 Bini EJ, Micale PL, Weinshel EH. Evaluation of the gastrointestinal tract in premenopausal women with iron deficiency anaemia. Am f Med 1998;105:281-6.

29 Tovey H, Godfrey JE, Lewin MR. A gastrectomy population: $25-30$ years on. Postgrad Med f 1990;66:450- 


\section{LETTERS TO THE EDITOR}

\section{Inflammation at the neo squamo- columnar junction in Barrett's oesophagus}

EDITOR,- - In the recent article entitled "Inflammation of the gastro-oesophageal junction (carditis) in patients with symptomatic gastro-oesophageal reflux disease: a prospective study" (Gut 1999;45:484-488), the authors determined that mucosal injury at the gastric cardia is highly localised to the region adjacent to the squamo-columnar junction in patients with gastro-oesophageal reflux disease (GORD). This is of particular interest to us in view of our recent work on the inflammatory response in Barrett's oesophagus. We have shown that while the Barrett's segment may be relatively devoid of inflammation, the neo squamo-columnar junction continues to excite an inflammatory reaction. ${ }^{1}$ These results were independent of patient medication $(\mathrm{n}=50, \mathrm{p}<0.05)$, similar to the study by Lembo et al.

Lembo et al suggest that carditis may be due to "wear and tear" at the gastrooesophageal junction as well as secondary to gastro-oesophageal reflux and Helicobacter pylori infection. Our similar findings in patients with Barrett's oesophagus suggest that the gastric and intestinal types of epithelium (either in normal stomach or in metaplastic oesophagus) may represent an adaptation to frequent exposure to refluxate. In contrast, the squamo-columnar junction is particularly susceptible to inflammation. It is interesting to speculate whether it is the proximal squamous oesophagus or the distal gastric/intestinal mucosa which excites the inflammatory response at the junction of these epithelia. In the study by Lembo et al, the biopsies containing squamous mucosa alone were not particularly inflamed; this suggests that it may be the interaction of cytokines generated from both the columnar and squamous epithelium in close proximity which are necessary to generate an inflammatory reaction. This may have implications for the strictures which occur in proximal Barrett's oesophagus.

R C FITZGERALD

Digestive Diseases Research Centre, St Barts and the Royal London School of Medicin and Dentistry, Turner Street, London E1 2AD, UK r.c.fitzgerald@mds.qmw.ac.uk

1 Fitzgerald R, Onwuegbusi B, Saeed I, et al. Differential degree of inflammation and cytokine expression in distal compared with proximal Barrett's oesophagus may explain site specific disease complications. Gastroenterology 1999; 116:A1762.

\section{Mother to child transmission of anti- $S$ cerevisiae mannan antibodies (ASCA) in non-IBD families}

EDIToR,-We enjoyed the recent study by Sutton and colleagues (Gut 2000;46:58-63) which confirmed our previous findings that elevated anti-S cerevisiae mannan antibodies (ASCA) are a familial trait in Crohn's disease. ${ }^{1}$ The lack of concordance in marital

Table 1 Distribution of anti-S cerevisiae mannan antibodies (ASCA) in 94 diabetic families

\begin{tabular}{|c|c|c|c|c|c|c|c|c|c|}
\hline \multirow{2}{*}{$\begin{array}{l}\text { Families } \\
\text { Children } \\
\text { per family }\end{array}$} & \multirow[b]{2}{*}{$n$} & \multicolumn{4}{|l|}{ Parents } & \multicolumn{4}{|l|}{ Children } \\
\hline & & $\begin{array}{l}\text { Total } \\
\text { number }\end{array}$ & $A S C A^{+}$ & $\begin{array}{l}\text { Father } \\
\text { ASCA }^{+}\end{array}$ & $\begin{array}{l}\text { Mother } \\
\text { ASCA }^{+}\end{array}$ & $\begin{array}{l}\text { Total } \\
\text { number }\end{array}$ & $A S C A^{+}$ & $\begin{array}{l}\text { Children } \\
\text { ASCA+ } A^{+} \text {born of } \\
A S C A^{+} \text {parents }\end{array}$ & $\begin{array}{l}\text { Children } \\
\text { ASCA } A^{+} \text {born of } \\
\text { ASCA } A^{-} \text {parents }\end{array}$ \\
\hline 1 & 22 & 44 & 5 & 4 & 1 & 22 & 0 & 0 & 0 \\
\hline 2 & 30 & 60 & 3 & 1 & 2 & 60 & 0 & 0 & 0 \\
\hline 3 & 34 & 68 & 5 & 1 & 4 & 102 & 4 & 4 & 0 \\
\hline 4 & 3 & 6 & 0 & 0 & 0 & 12 & 0 & 0 & 0 \\
\hline 5 & 3 & 6 & 1 & 0 & 1 & 15 & 1 & 1 & 0 \\
\hline 6 & 0 & 0 & 0 & 0 & 0 & 0 & 0 & 0 & 0 \\
\hline 7 & 2 & 4 & 1 & 0 & 1 & 14 & 3 & 3 & 0 \\
\hline Total & 94 & 188 & 15 & 6 & 9 & 225 & 8 & 8 & 0 \\
\hline
\end{tabular}
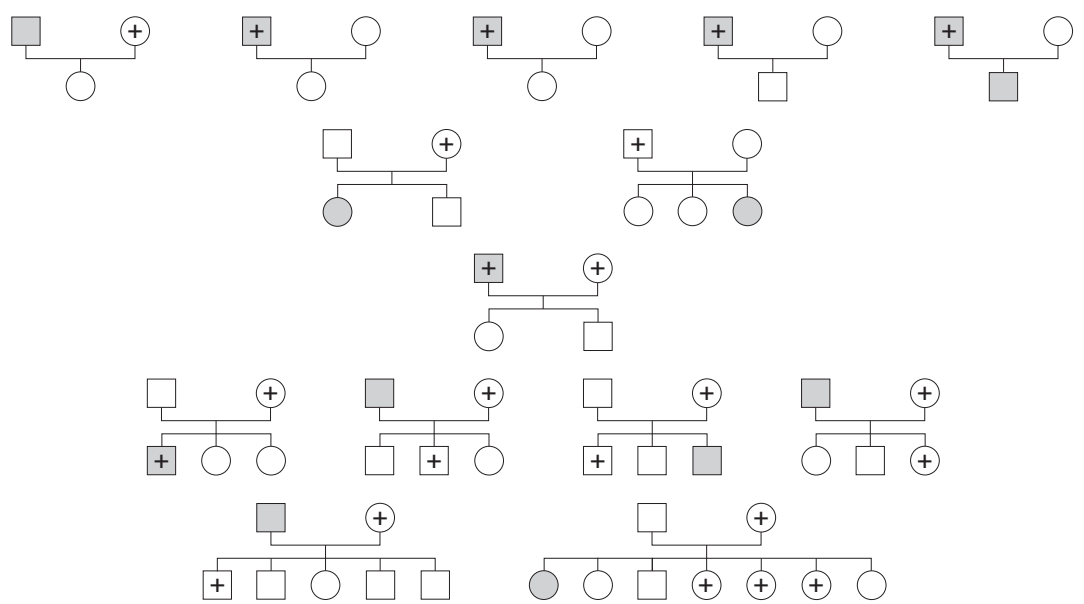

Figure 1 Pedigrees of the 14 diabetic families with at least one ASCA positive subject. +, presence of ASCA; shaded box, patient with diabetes.

pairs indicated that familiality may be due to either a genetic factor or childhood environmental exposure. ASCA are present in $50-60 \%$ of patients with Crohn's disease, in $10-15 \%$ of patients with ulcerative colitis, and in $2-5 \%$ of control subjects. ${ }^{2-5}$ To gain more information on concordance for ASCA among family members, we studied ASCA distribution in non-inflammatory bowel disease (IBD) families.

A total of 413 serum samples were collected from 94 diabetic families (table 1). One patient per family had type I diabetes. ASCA were detected by ELISA as previously described. ${ }^{3}$ Distribution of ASCA is given in fig 1 and table 1 . Twenty three subjects (5.6\%) were ASCA positive: six had diabetes and 14 were healthy. ASCA positive subjects were distributed within only 14 of 96 families. In seven of these families only one subject (parent) was positive for ASCA. The remaining 16 ASCA positive subjects clustered within seven families. All ASCA positive children were born of an ASCA positive mother. These results show that familiality of ASCA occurs independently of Crohn's disease and suggest vertical transmission of the marker from mother to child. Whether this is related to the observed higher risk of Crohn's disease transmission from mother to child than from father to child ${ }^{6}$ is unknown. Further work is needed to assess if the presence of ASCA may predict an increased risk of Crohn's disease in offspring.

D POULAIN

B SENDID

I FAJARDY

PM DANZE JF COLOMBEL

Laboratoire de Mycologie Fondamentale et Appliquée, Equipe INSERM 99-15,
Laboratoire de Recherche sur les Maladies Inflammatoires Intestinales (CRI 4U004B), Unité fonctionnelle de génotypage HLA, $C H$ et $U$ Lille, 59037, France

Correspondence to: J F Colombel.

jfcolombel@chru-lille.fr

1 Sendid B, Quinton JF, Charrier G, et al. Anti-Saccharomyces cerevisiae mannan antibodies in familial Crohn's disease. $\mathrm{Am} \mathcal{F}$ Gastroenterol 1998;93:1306-10.

2 Main J, McKenzie H, Yeaman GR, et al. Antibody to Saccharomyces cerevisiae (bakers' yeast) in Crohn's disease. BMF 1988;297: $1105-6$

3 Sendid B, Colombel JF, Jacquinot PM, et al. Specific antibody response to oligomannosidic Specific antibody response to oligomannosidic
epitopes in Crohn's disease. Clin Diagn Lab epitopes in Crohn's disea

4 Quinton JF, Sendid B, Reumaux D, et al. Anti-Saccharomyces cerevisiae mannan antibodies combined with antineutrophil cytoplasmic autoantibodies in inflammatory bowel disease: prevalence and diagnostic role. Gut 1998;42:788-91

5 Ruemmele FM, Targan SR, Levy G, et al. Diagnostic accuracy of serological assays in pediatric inflammatory bowel disease. Gastroenterology $1998 ; \mathbf{1 1 5}: 822-9$.

6 Akolkar PN, Gulwani-Akolkar B, Heresbach D, et al. Differences in risk of Crohn's disease in offspring of mothers and fathers with inflam matory bowel disease. Am f Gastroenterol 1997; 92:2241-4.

Reply

EDITOR,- We were interested by the letter of Poulain and colleagues on our paper (Gut 2000;46:58-63) and their data on familiality of this trait in non-inflammatory bowel disease (IBD) families, and evidence for mother to child transmission of anti-S cerevisiae mannan antibody (ASCA) expression. We have now re-analysed our own data set to our findings on this issue (table 1). We 
observed a strong correlation for both the mother-child and father-child pairs. This differs from Poulain and colleagues who only observed vertical transmission from mother to child. The difference may relate to distinct subject populations. Poulain evaluated a nonIBD group (diabetic families) whereas we evaluated clinically unaffected relatives from families with Crohn's disease (CD) probands. We also note that our analysis used statistical methods for quantitative data (antibody levels) which enhances statistical assessment, particularly when limited by small data sets. Poulain's analysis compares qualitative data (seropositive or seronegative), and the small number of relevant pairs may be insufficient to support their conclusion statistically. We agree with them on the need to assess the possibility that ASCA may predict increased risk for $\mathrm{CD}$ in unaffected individuals.

Table 1 Correlation coefficients for IgG anti-S cerevisiae mannan antibodies (ASCA) among 333 first degree relatives without evidence of Crohn's disease

\begin{tabular}{ll}
\hline Type of pairs & $\operatorname{IgGASCA}$ \\
\hline Mother-daughter & 0.135 \\
Mother-son & 0.483 \\
Father-daughter & 0.423 \\
Father-son & 0.404 \\
\hline
\end{tabular}

J BRAUN

CL SUTTON

HY YANG

J ROTTER

Department of Pathology and Lab Medicine, UCLA and Division of Medical Genetics and IBD Research Center, Cedars-Sinai Medical Center, Los Angeles, CA 90095-1732, USA jbraun@mednet.ucla.edu

\section{BOOK REVIEW}

MEDLINE: A Guide to Effective Searching. Edited by Katcher BS (Pp147; $\$ 29.00)$ US: Ashbury Press, 1999. ISBN 0 967344506 .

Many specialist registrars embarking on a period of research are faced with the task of writing grant proposals requiring a comprehensive understanding of a subject, but often begin from a point of relative ignorance. The first port of call for many will be to delve into MEDLINE, a vast bibliographic database indexing over 4000 journals. Now freely accessible through the internet, MEDLINE can be used to search for subject information in what appears at the outset to be a deceptively simplistic way. However, as many users of this database will know, finding the exact information you require can prove both time consuming and unrewarding, generating large amounts of inappropriate references to the subject in question. This is a reflection not of the inadequacies of the MEDLINE database but of the method of searching being employed by the researcher.

Successful searching requires knowledge of the vocabulary and grammar developed for querying such a database. Brian Katcher's book provides the reader with such know- ledge, relevant not only to MEDLINE, but bibliographic databases organised in a similar format, sparing the reader from many hours of fruitless searching. The opening chapter provides a brief historical introduction as to how MEDLINE was conceived and the way in which data are indexed, a key to understanding the most efficient way of extracting relevant information.

The novice user of MEDLINE will in most cases begin searching using text or keywords, often generating a list of references that is simply too long to read. Chapter two takes the reader through the different fields or indexes that can be used when searching and details how the long list of references obtained can be systematically fine tuned using combinations of these. Particular emphasis is placed on the usefulness of Medical Subject Headings (MeSH), an underlying theme of the book, as an initial means of searching, and useful hints are given to direct the reader to the $\mathrm{MeSH}$ headings most appropriate to the information they require. The book concludes by setting out some standard approaches to effective searching using information learned in an initial search strategy to direct subsequent avenues of enquiry. In addition it gives useful hints on the framing of questions in order to get results most appropriate to the topic of enquiry.

MEDLINE contains a vast array of information and perhaps Brian Katcher's most useful tip is to start with books, pencil, and paper. His book is an informative read, most useful to those who are relative novices to using MEDLINE. It is presented in an easy style and without doubt will guide the reader to more "effective searching" of bibliographic databases.

J A TIBBLE

\section{NOTES}

Sir Francis Avery Jones British Society of Gastroenterology Research Award 2001

Applications are invited by the Education Committee of the British Society of Gastroenterology who will recommend to Council the recipient of the 2001 Award. Applications (TWENTY COPIES) should include:

- A manuscript (2 A4 pages ONLY) describing the work conducted

- A bibliography of relevant personal publications

- An outline of the proposed content of the lecture, including title

- A written statement confirming that all or a substantial part of the work has been personally conducted in the UK or Eire.

Entrants must be 40 years old or less on 31 December 2000 but need not be a member of the Society. The recipient will be required to deliver a 30 minute lecture at the Annual meeting of the Society in Glasgow in March 2001. Applications (TWENTY COPIES) should be made to the Honorary Secretary, British Society of Gastroenterology, $3 \mathrm{St}$ Andrews Place, London NW1 $4 \mathrm{LB}$ by 1 December 2000 .
British Society of Gastroenterology Hopkins Endoscopy Prize 2001

Applications are invited by the Endoscopy Committee of the British Society of Gastroenterology who will recommend to the Council the recipient of the 2001 Award. Applications (TEN COPIES) should include:

- A manuscript (2 A4 pages ONLY) describing the work conducted

- A bibliography of relevant personal publications

- An outline of the proposed content of the lecture, including title

- A written statement confirming that all or a substantial part of the work has been personally conducted in the UK or Eire.

An applicant need not be a member of the Society. The recipient will be required to deliver a 20 minute lecture at the Annual meeting of the Society in Glasgow in March 2001. Applications (TEN COPIES) should be made to the Endoscopy Section Secretary, British Society of Gastroenterology, $3 \mathrm{St}$ Andrews Place, London NW1 4LB by 1 December 2000.

\section{American College of Gastroenterology 2001 International GI Training Grants Programme}

The ACG International GI Training (IGT) Grant Programme provides funding for clinical or clinical research training in gastroenterology and hepatology so that an individual can acquire or develop new cognitive knowledge or a technical skill. This newly acquired knowledge or skill would then be used to improve patient care in the applicant's geographic area. Physicians outside of the United States and Canada are eligible to apply. At least one fellowship with a maximum of $\$ 10000$ per IGT fellowship will be awarded during 2001, for a training period of not less than six months. Awards will be made by a special committee of the ACG and will be based upon the applicant's credentials, the merit of the proposed training by the selected host training centre and the potential for enhancing the field of gastroenterology in the applicant's home country. Application forms can be obtained from the ACG administrative office: 4900B South 31st Street, Arlington, Virginia 22206-1656. Tel: +1 703820 7400; fax: +1 703931 4520; website: www.acg.gi.org. Deadline for submission of application is 1 April 2001.

\section{3rd European Federation of Autonomic} Societies (EFAS)

The third European Federation of Autonomic Societies (EFAS) meeting in conjunction with the annual meeting of the sections "Autonomic nervous system" of the German Neurological Society, "Diabetes and Nervous System" of the German Neurological Society, and "Autonomic Nervous System" at the University of Erlangen-Nuremberg, Germany, will be held in Erlangen, Germany on 26-28 April 2001. Abstract deadline: 20 December 2000. Further information: Professor Dr M J Hilz, Department of Neurology, University or Erlangen-Nuremberg, Schwabachanlage 6, D-91054 Erlangen, Germany. Tel: +49 0131 8534444; fax: +49 9131 8534328; website: 
www.neurologie.med.uni-erlangen.de/ oeffentliche_Veranstaltungen.htm

Cleveland Clinic Florida's

\section{Gastroenterology Update 2001}

Cleveland Clinic Florida will be sponsoring a postgraduate course entitled "Gastroenterology Update 2001 " to be held on 10-11 February 2001 in Fort Lauderdale, Florida,

USA. Further information: Sally Jagelman, Manager of Continuing Medical Education, Cleveland Clinic Florida, 3000 West Cypress Creek Road, Fort Lauderdale, FL 33309 , USA. Tel: +1 954978 5539; fax: +1954978 5056; email: jagelms@ccf.org

GI malignancies can be prevented and treated: from the bench to the bedside

This international meeting will be held on 14-17 February 2001 in Jerusalem and the Dead Sea, Israel. Further information: Mari- lyne Katz, Secretariat, GI Malignancies, Target Tours, PO Box 29041, Tel Aviv 61290, Israel. Tel: +972 3 5175150; fax: +972 3 5175155; email: gi@targetconf.com

\section{Redefining Priorities in}

Gastroenterology

This congress will be held on 11-14 April 2001 in Monte Carlo, Italy. It will be chaired by Professor Massimo Crespi (Rome, Italy) and Professor Eammon Quigley (Cork, Ireland). Further information: Maddalena Massaro, Project Leader, AISC-AIM Group, Via A Ristori 38, 00187 Rome, Italy. Tel: +39 06 809681; fax: +39 06 80968229; email: m.massaro@aisc.it.

\section{Gastroenterology and Endotherapy: XIXth European Workshop}

This course, to introduce the experienced gastroenterologist to the growing field of therapeutic endoscopy, will be held on 18-20 June 2001 in Brussels, Belgium. Further information: Mrs Nancy Beauprez, Gastroenterology Department, Erasme Hospital, Route de Lennik 808, B-1070 Brussels. Tel: +320255549 00; fax: +320255549 01; email: beauprez@ulb.ac.be

\section{CORRECTION}

The authors of Guidelines for the management of iron deficiency anaemia (Gut 2000;46(suppl IV)) would like to correct an error they made. In section 2.2 , second paragraph, the wrong unit was used for ferritin. Instead of $\mu \mathrm{g} / \mathrm{dl}$, it should have been $\mu \mathrm{g} / 1$ (or $\mathrm{ng} / \mathrm{ml}$ ). The authors apologise for any confusion this may have caused.

8th United European Gastroenterology Week

The UEGW abstract book (Gut 2000;47(suppl III)) has again been produced as a CD-ROM and can be found attached to the inside back cover of this issue. 